\title{
ON MAXIMAL CONVEX LATTICE POLYGONS INSCRIBED IN A PLANE CONVEX SET
}

\author{
BY \\ IMRE BÁRÁNY* \\ Rényi Institute, Hungarian Academy of Sciences \\ POB 127, 1364 Budapest, Hungary \\ and \\ Department of Mathematics, University College London \\ Gower Street, London, WC1E 6BT, UK \\ e-mail: barany@renyi.hu \\ AND \\ Maria Prodromou \\ Department of Mathematics, University College London \\ Gower Street, London, WC1E 6BT, UK \\ e-mail: maria@math.ucl.ac.uk
}

\begin{abstract}
Given a convex compact set $K \subset \mathbb{R}^{2}$ what is the largest $n$ such that $K$ contains a convex lattice $n$-gon? We answer this question asymptotically. It turns out that the maximal $n$ is related to the largest affine perimeter that a convex set contained in $K$ can have. This, in turn, gives a new characterization of $K_{0}$, the convex set in $K$ having maximal affine perimeter.
\end{abstract}

\section{Introduction}

Assume $K \subset \mathbb{R}^{2}$ is a fixed convex body, that is a convex compact set with nonempty interior. Let $\mathbb{Z}^{2}$ denote the (usual) lattice of integer points and write $\mathbb{Z}_{t}=\frac{1}{t} \mathbb{Z}^{2}$. This is a shrunken copy of $\mathbb{Z}^{2}$ when $t$ is large. A convex $\mathbb{Z}_{t}$-lattice

* Supported by Hungarian National Foundation Grants T 046246 and 'T 037846. Received October 8, 2004 
$n$-gon is, by definition, a convex polygon with exactly $n$ vertices each belonging to the lattice $\mathbb{Z}_{t}$. Define

$m\left(K, \mathbb{Z}_{t}\right)=\max \left\{n:\right.$ there is a convex $\mathbb{Z}_{t}$-lattice $n$-gon contained in $\left.K\right\}$.

In other words, $m\left(K, \mathbb{Z}_{t}\right)$ is the maximal number of $\mathbb{Z}_{t}$-lattice points in $K$ in convex position. In this paper we determine the asymptotic behavior of $m\left(K, \mathbb{Z}_{t}\right)$ (as $t \rightarrow \infty$ ). We now state the main result. Let $A(K)$ denote the supremum of the affine perimeter of all convex sets $S \subset K$. (For the definition and properties of affine perimeter, see Section 2.)

TheOREM 1.1: Under the above conditions,

$$
\lim _{t \rightarrow \infty} t^{-2 / 3} m\left(K, \mathbb{Z}_{t}\right)=\frac{3}{(2 \pi)^{2 / 3}} A(K) .
$$

Let $\operatorname{AP}(S)$ denote the affine perimeter of a convex set $S \subset \mathbb{R}^{2}$. It is shown in [Bá97] (see also Theorem 3.1 below) that there is a unique $K_{0} \subset K$ with $\mathrm{AP}\left(K_{0}\right)=A(K)$. This unique $K_{0}$ has the interesting "limit shape" property (see [Bá95] and [Bá97]) that the overwhelming majority of the convex $\mathbb{Z}_{t}$-lattice polygons contained in $K$ are very close to $K_{0}$ in the Hausdorff distance. This property extends to the above case as well:

ThEOREM 1.2: Let $Q_{t}$ denote any maximizer in the definition of $m\left(K, \mathbb{Z}_{t}\right)$. Then

where dist denotes the Hausdorff distance.

$$
\lim _{t \rightarrow \infty} \operatorname{dist}\left(Q_{t}, K_{0}\right)=0
$$

The problem of estimating $m\left(K, \mathbb{Z}_{t}\right)$ has a long history. Jarnik proved in [Ja25] that on a strictly convex curve of length $\ell$ in the plane there can be at most

$$
\frac{3}{\sqrt[3]{2 \pi}} \cdot \ell^{2 / 3}(1+o(1))
$$

lattice points and this estimate is best possible. When the strictly closed curve is the circle of radius $r$, Jarník's estimate gives that a convex polygon contained in this circle has at most $3 \sqrt[3]{2 \pi} r^{2 / 3}(1+o(1))$ vertices. The same bound follows from Theorem 1.1 as well.

Andrews [An63] showed that a convex lattice polygon $P$ has at most $c(\text { Area } P)^{1 / 3}$ vertices, where $c>0$ is a universal constant. The smallest known value of $c$ is $\left(8 \pi^{2}\right)^{1 / 3}<5$, which follows from an inequality of Rényi and Sulanke [RS63] (see [Ra93]). This implies in the $K, \mathbb{Z}_{t}$ setting that

$$
m\left(K, \mathbb{Z}_{t}\right) \leq 5 t^{2 / 3}(\text { Area } K)^{1 / 3},
$$




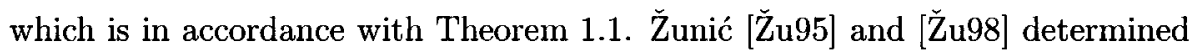
$m\left(K, \mathbb{Z}_{t}\right)$ when $K$ is the unit square, and the limiting shape of the maximizer.

Remark 1.1: The lattice points on the curve giving the extremum form a convex lattice polygon, which is called Jarník's polygon. It is clear that its edges are "short" primitive vectors. We will see this phenomenon in the proof of Theorems 1.1 and 2.1 .

Remark 1.2: Actually, Andrews [An63] proves much more. Namely, a convex lattice polytope $P \subset \mathbb{R}^{d}$ with nonempty interior can have at most $c(\operatorname{vol} P)^{(d-1) /(d+1)}$ vertices where the constant $c>0$ depends on dimension only.

\section{Affine perimeter}

Let $\mathcal{C}$ denote the set of convex bodies in $\mathbb{R}^{2}$, that is, compact convex sets with nonempty interior. Given $S \in \mathcal{C}$, choose a subdivision $x_{1}, \ldots, x_{n}, x_{n+1}=x_{1}$ of the boundary $\partial S$ and lines $\ell_{i}, i=1, \ldots, n$ supporting $S$ at $x_{i}$. Denote by $y_{i}$ the intersection of $\ell_{i}, \ell_{i+1}$ and by $T_{i}$ the triangle conv $\left\{x_{i}, y_{i}, x_{i+1}\right\}$ (and also its area). The affine perimeter $A P(S)$ of $S$ is defined as

$$
A P(S)=2 \lim \sum_{i=1}^{n} \sqrt[3]{T_{i}}
$$

where the limit is taken over a sequence of subdivisions with

$$
\max _{1 \ldots, n}\left|x_{i+1}-x_{i}\right| \rightarrow 0
$$

The existence of the limit and its independence of the sequence chosen follow from the fact, implied by the inequality in (2.4) below, that $\sum_{i=1}^{n} \sqrt[3]{T_{i}}$ decreases as the subdivision is refined. Therefore,

$$
A P(S)=2 \inf \sum_{i=1}^{n} \sqrt[3]{T_{i}}
$$

Note that the affine perimeter of a polygon is zero.

The same definition applies for a compact convex curve $\Gamma$ : a subdivision $x_{1}, \ldots, x_{n+1}$ on $\Gamma$, together with the supporting lines at $x_{i}$ define the triangles $T_{1}, \ldots, T_{n}$, and $A P(\Gamma)$ is the infimum of $2 \sum_{i=1}^{n} \sqrt[3]{T_{i}}$. Alternatively, given unit vectors $d_{1}, \ldots, d_{n+1}$ (in clockwise order on the unit circle), there is a subdivision $x_{1}, \ldots, x_{n+1}$ on $\Gamma$ with tangent line $\ell_{i}$ at $x_{i}$ which is orthogonal to $d_{i}$. The 
subdivision defines triangles $T_{1}, \ldots, T_{n}$, and

$$
A P(\Gamma)=2 \inf \sum_{i=1}^{n} \sqrt[3]{T_{i}}
$$

where now the infimum is taken over all $n$ and all choices of unit vectors $d_{1}, \ldots, d_{n+1}$. Note that the triangles $T_{i}$ are determined by $\Gamma$ and $d_{1}, \ldots, d_{n+1}$ uniquely (unless $d_{i}$ is orthogonal to a segment contained in $\Gamma$, in which case we can take the midpoint of this segment for $x_{i}$ ). We will call them the triangles induced by directions $d_{1}, \ldots, d_{n+1}$ on $\Gamma$.

We mention the following properties of the map $A P: \mathcal{C} \rightarrow \mathbb{R}$ that will be used later.

(2.1) $A P(L S)=(\operatorname{det} L)^{1 / 3} A P(S)$, for $L: \mathbb{R}^{2} \rightarrow \mathbb{R}^{2}$ linear.

(2.2) If the boundary of $S$ is twice differentiable, then $A P(S)=\int_{\partial S} \kappa^{1 / 3} d s=$ $\int_{0}^{2 \pi} r^{2 / 3} d \phi$, where $\kappa$ is the curvature and $r$ the radius of curvature at the boundary point with outer normal vector $u(\phi)=(\cos \phi, \sin \phi)$.

(2.3) Given a triangle $T=\operatorname{conv}\left\{p_{0}, p_{1}, p_{2}\right\}$, let $D=D(T)$ be the unique parabola which is tangent to $p_{0} p_{1}$ and $p_{1} p_{2}$ at $p_{0}$ and $p_{2}$, respectively. Among all convex curves connecting $p_{0}$ and $p_{2}$ within the triangle $T$, the arc of the parabola $D$ is the unique one with maximal affine length, and $A P(D)=2 \sqrt[3]{T}$. We call $D$ the special parabola in $T$.

(2.4) In the above triangle $T$ let $q_{1}$ and $q_{2}$ be points on the side $p_{0} p_{1}$ and $p_{1} p_{2}$, resp., and let $p_{3}$ be a point on $q_{1} q_{2}$. Writing $T_{1}$ and $T_{2}$ for triangles $p_{0} q_{1} p_{3}$ and $p_{3} q_{2} p_{2}$ we have (see Figure 1)

$$
\sqrt[3]{T} \geq \sqrt[3]{T_{1}}+\sqrt[3]{T_{2}}
$$

Moreover, equality holds if and only if $q_{1} q_{2}$ is tangent to the parabola $D$ at the point $p_{3}$ (see [Bl23]). 


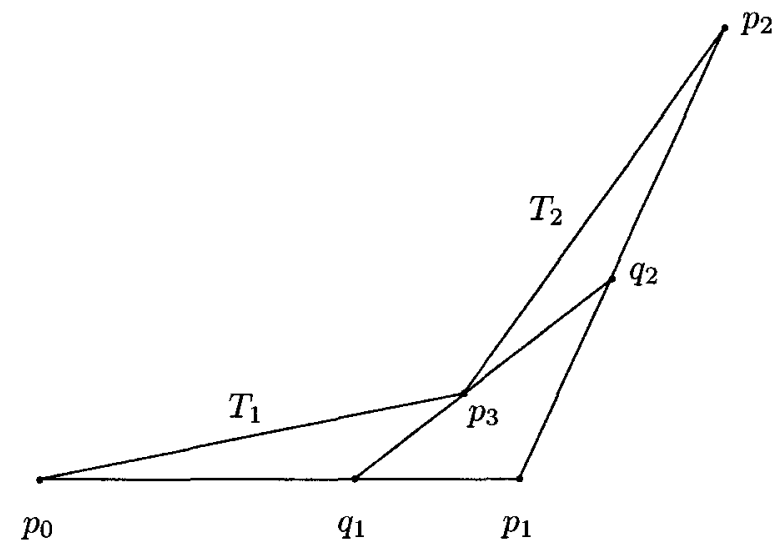

Figure 1.

It is clear from the definition that, for a polygon $K, A P(K)=0$. This shows further that the map $A P: \mathcal{C} \rightarrow \mathbb{R}$ is not continuous $(\mathcal{C}$ is equipped with the Hausdorff metric). It is known (see [Lu91], for instance), however, that it is upper semicontinuous.

The following theorem will be used for the proof of the main theorems. It is similar, in spirit, to a result of Vershik [Ve94]. Assume $\Gamma$ is a compact convex curve in the plane. For $\varepsilon>0$, the $\varepsilon$-neighbourhood of $\Gamma$ will be denoted by $U_{\varepsilon}(\Gamma)$. Let $m\left(\Gamma, \varepsilon, \mathbb{Z}_{t}\right)$ denote the maximum number of vertices that a convex $\mathbb{Z}_{t}$-lattice curve lying in $U_{\varepsilon}(\Gamma)$ can have.

THEOREM 2.1: Under the above conditions,

$$
\lim _{\varepsilon \rightarrow 0} \lim _{t \rightarrow \infty} t^{-2 / 3} m\left(\Gamma, \varepsilon, \mathbb{Z}_{t}\right)=\frac{3}{(2 \pi)^{2 / 3}} A P(\Gamma) .
$$

For the proof of Theorem 2.1 we will need the following fact, which is a consequence of the upper semicontinuity of the affine perimeter.

Proposition 2.1: For every compact convex curve $\Gamma$ and for every $\eta>0$ there exist $\varepsilon>0$, integer $n$, and unit vectors $d_{1}, \ldots, d_{n+1}$ such that for every compact convex curve $\Gamma^{\prime} \subset U_{\varepsilon}(\Gamma)$ the triangles $T_{1}, \ldots, T_{n}$ induced by $d_{1}, \ldots, d_{n+1}$ on $\Gamma^{\prime}$ satisfy

$$
2 \sum_{i=1}^{n} \sqrt[3]{T_{i}} \leq A P(\Gamma)+\eta
$$


Proof: This is quite simple: assuming the contrary one finds a $\Gamma^{\prime} \subset U_{\varepsilon}(\Gamma)$ for every $\varepsilon>0$ with $A P\left(\Gamma^{\prime}\right)>A P(\Gamma)+\eta$, contradicting the upper semicontinuity of the functional $A P$.

\section{Maximal affine perimeter}

Let $\mathcal{C}(K)=\{S \in \mathcal{C}, S \subset K\}$. Define $A(K)=\sup \{A P(S), S \in \mathcal{C}(K)\}$. We will need the following result from [Bá97].

Theorem 3.1: For every $K \in \mathcal{C}$ there exists a unique $K_{0} \in \mathcal{C}(K)$ such that $A P\left(K_{0}\right)=A(K)$.

The function $A: \mathcal{C} \rightarrow \mathbb{R}$ is continuous. (We omit the simple proof.) Theorem 3.1 shows that there is a mapping $F: \mathcal{C} \rightarrow \mathcal{C}$ given by $F(K)=K_{0}$. $F$ is affinely equivariant, that is, for a nondegenerate affine map $L: \mathbb{R}^{2} \rightarrow \mathbb{R}^{2}$, $F(L K)=L F(K)$.

Proposition 3.1: $F: \mathcal{C} \rightarrow \mathcal{C}$ is continuous.

Proof: The proof is simple: assume $K_{n} \rightarrow K$. Choose a convergent subsequence of $F\left(K_{n}\right)$; its limit, $K^{*}$ say, is contained in $K$. The upper semicontinuity of $A P$ and the continuity of $A$ implies that

$$
A P\left(K^{*}\right) \geq \lim \sup A P\left(F\left(K_{n}\right)\right)=\lim A\left(K_{n}\right)=A P(F(K)),
$$

and $K^{*}=F(K)=K_{0}$ follows from the unicity of $K_{0}$.

The unique $F(K)=K_{0}$ has interesting properties as well. Clearly, $\partial K_{0} \cap \partial K \neq \emptyset$, as otherwise a slightly enlarged copy of $K_{0}$ would be contained in $K$ and have larger affine perimeter. So, $\partial K_{0} \backslash \partial K$ is the union of countably many arcs, called free arcs.

(3.1) Each free arc is an arc of a parabola whose tangents at the end points are tangent to $K$ as well.

(3.2) The boundary of $K_{0}$ contains no line segment.

The last statement is made quantitative in [Bá99]: Assume Area $K=1$. Assume further that the maximal area ellipsoid inscribed in $K_{0}$ is a circle. This can be reached using a suitable area preserving affine transformation.

(3.3) Under these conditions the radius of curvature at each point on the boundary of $K_{0}$ is at most 240 . 
What we get from the proofs of the main theorems is a characterization of $K_{0}$. For $C \in \mathcal{C}$ the barycenter (or center of gravity) of $C$ is defined by

$$
b(C)=\frac{1}{\text { Area } C} \int_{x \in C} x d x .
$$

(Here, $x$ is a vector and $d x$ is integration on $C$.) Define $\mathcal{C}_{0}$ as the collection of all $C \in \mathcal{C}$ with $b(C)=0$. Fix $C \in \mathcal{C}_{0}$ and let $u \in S^{1}$ be a unit vector. The radial function, $\rho(u)=\rho_{C}(u)$, is, as usual (cf. [Sch93]), defined as

$$
\rho_{C}(u)=\max \{t>0: t u \in C\}
$$

The condition $\int_{C} x d x=0$ translates (via a change of variables to polar coordinates) to

$$
\int_{S^{1}} \rho(u)^{3} d u=0
$$

here, $d u$ denotes vector integration on $S^{1}$. By Minkowski's classical theorem (see [Sch93]), there is a unique (up to translation) convex body $C^{*}=G(C)$ whose radius of curvature in direction $u$ is exactly $R(u)=\frac{1}{3} \rho^{3}(u)$. The following characterization theorem describes the sets $F(K)$ when $K \in \mathcal{C}$.

Theorem 3.2: For each $K \in \mathcal{C}$, there is a unique $C \in \mathcal{C}_{0}$ such that $K_{0}$ is a translated copy of $G(C)=C^{*}$. Moreover, for every $C \in \mathcal{C}_{0}$ the set $G(C)=$ $C^{*} \in \mathcal{C}$ satisfies $F\left(C^{*}\right)=C^{*}$.

This theorem immediately implies the following result.

Corollary 3.1: Assume $K \in \mathcal{C}$. Then $F(K)=K$ holds if and only if $K$ has well-defined and continuous radius of curvature $R\left(u\right.$ ) (for each $u \in S^{1}$ ) and $\sqrt[3]{3 R(u)}$ is the radial function of a convex set $C \in \mathcal{C}_{0}$.

Two sets $K_{1}, K_{2} \in \mathcal{C}$ are equivalent if they are translates of each other. Write $\mathcal{K}$ for the set of equivalence classes in $\{F(K): K \in \mathcal{C}\}$. The two theorems above show that the map $G: \mathcal{C}_{0} \rightarrow \mathcal{K}$ is one-to-one. It can be shown that the map $G: \mathcal{C}_{0} \rightarrow \mathcal{K}$ is continuous in both directions, but we won't need this fact here.

Theorem 3.2 implies the following strengthening of (3.3):

COROLlARY 3.2: For any $K \in \mathcal{C}$, there is a nondegenerate linear transformation $L: \mathbb{R}^{2} \rightarrow \mathbb{R}^{2}$ such that the radius of curvature $R(u)$ of $F(L K)=(L K)_{0}$ at any point of its boundary satisfies

$$
\frac{1}{3} \leq R(u) \leq \frac{8}{3}
$$


Remark 3.1: Theorem 3.2 and Corollary 3.1 may extend to higher dimensions. Unfortunately, the unicity of the maximal affine surface area convex set contained in a fixed convex body in $\mathbb{R}^{d}$ for $d>2$ is not known. We hope to return to these questions in the near future.

\section{Large triangles}

We are interested in the maximal convex $\mathbb{Z}_{t}$-lattice polygons inscribed in a convex body $K$, when $t$ is large. This is the same as considering the maximal $\mathbb{Z}^{2}$-lattice polygons inscribed in the blown-up copy $t K$ of $K$. Theorems 1.1 and 1.2 show that any such maximizer is very close to the subset $K_{0}$ of $K$ with maximal affine perimeter. As we saw earlier, the boundary of this body $K_{0}$ is the union of countably many parabolic arcs whose tangents at the end points are tangent to $K$ as well. These tangent lines will define our "large" triangles. We will be interested in finding the set of vectors that will build up the arc of $Q_{t}$ within each such triangle $T$. We shall prove that each large triangle naturally gives rise to a "small" triangle $\Delta$, so that the edges of the arc of the maximizer $Q_{t}$ within $T$ are primitive vectors in $\Delta$. These connections will become clear in the sections to come.

We now proceed with a result about large triangles. We start with a definition which is slightly more general than necessary.

Definition 1: Let $T=\operatorname{conv}\left\{p_{0}, p_{1}, p_{2}\right\}$ be a (non-degenerate) triangle in $\mathbb{R}^{2}$. A convex lattice chain within $T$ (from the side $\left[p_{0}, p_{1}\right]$ to the side $\left[p_{1}, p_{2}\right]$ ) is a sequence of points $x_{0}, \ldots, x_{n}$ such that

(i) the points $p_{0}, x_{0}, \ldots, x_{n}, p_{2}$ are in convex position,

(ii) $z_{i}=x_{i}-x_{i-1} \in \mathbb{Z}^{2}$, for each $i=1, \ldots, n$.

We call $n$ the length of this convex lattice chain.

Define $m(T)$ as the maximal length that a convex lattice chain within $T$ can have. For simpler writing we denote the area of $T$ by the same letter $T$.

Assume now that $a, b \in \mathbb{R}^{2}$ are two non-parallel vectors and $t_{1}, t_{2}$ are almost equal and large values. Setting $p_{1}-p_{0}=t_{1} a$ and $p_{2}-p_{1}=t_{2} b$ gives the "large" triangle $T$.

Theorem 4.1: Assume $t_{1}, t_{2} \rightarrow \infty$ with $t_{1} / t_{2} \rightarrow 1$. Then

$$
\lim m(T) \cdot T^{-1 / 3}=\frac{6}{(2 \pi)^{2 / 3}} .
$$

Clearly, it suffices to show this when $t_{1}=t_{2}=t$ and $t \rightarrow \infty$ and this is what we are going to do in Section 6 . 
We will need this result in the $\mathbb{Z}_{t}$ setting as well. So given a triangle $T$ in the plane, we define $m^{*}\left(T, \mathbb{Z}_{t}\right)$ as the length of a maximal $\mathbb{Z}_{t}$-lattice chain from vertex $p_{0}$ to vertex $p_{2}$ within $T$. The previous theorem states that

$$
\lim t^{-2 / 3} m^{*}\left(T, \mathbb{Z}_{t}\right)=\frac{6 \sqrt[3]{T}}{(2 \pi)^{2 / 3}}
$$

Now let $Q_{t}$ be a maximal $\mathbb{Z}_{t}$-lattice chain in $T$ (from $p_{0}$ to $p_{2}$ ). Recall the definition of the special parabola $D(T)$ from (2.3).

THEOREM 4.2: Under the above conditions,

$$
\lim \operatorname{dist}\left(Q_{t}, D(T)\right)=0
$$

This result follows from Theorem 1.2 as well. The short proof given in section 7 shows the close connection between maximal convex lattice chains and inequality (2.4).

From the proof of Theorem 4.1 we will be able to give a simple construction of a convex $\mathbb{Z}_{t}$-lattice curve in the triangle $T$ which is almost maximal and is very close to the parabola arc $D(T)$. This construction will be used in the characterization Theorem 3.2.

Remark 4.1: It would be interesting to understand the behavior of $m(T)$, for general triangles, when $T \rightarrow \infty$. Write $w(T)$ for the lattice width of the triangle $T$, that is

$$
w(T)=\min _{w \in \mathbb{Z}^{2} \backslash\{0\}} \max \{w(x-y): x, y \in T\} .
$$

If $w \in \mathbb{Z}^{2}$ is the lattice width direction of $T$, then the lattice points in any translated copy of $T$ are contained in $\lceil w(T)\rceil$ consecutive lattice lines. Each such line contains at most two vertices from a convex lattice chain. Thus,

$$
m(T) \leq 2\lceil w(T)\rceil<2 w(T)+2 .
$$

So, if $w(T)$ is much smaller than $T^{1 / 3}$, then the asymptotic estimate

$$
m(T) \approx \frac{6}{(2 \pi)^{2 / 3}} \sqrt[3]{T}
$$

dictated by Theorem 4.1 is no longer valid. 


\section{Small triangles}

Assume now that $u, v \in \mathbb{R}^{2}$ are non-parallel vectors. Define the triangle $\Delta$ as

$$
\Delta=\operatorname{conv}\{0, u, v\} .
$$

Its area is also denoted by $\Delta$ and its lattice width by $w(\Delta)$. We write $\mathbb{P}$ for the set of primitive vectors in $\mathbb{Z}^{2}$,

$$
\mathbb{P}=\left\{(x, y) \in \mathbb{Z}^{2}: \operatorname{gcd}(x, y)=1\right\} .
$$

We will need to know the size of $\mathbb{P} \cap \Delta$. Since the density of $\mathbb{P}$ in $\mathbb{Z}^{2}$ is asymptotically $6 / \pi^{2}$, one would expect

$$
|\mathbb{P} \cap \Delta| \approx \frac{6}{\pi^{2}} \Delta .
$$

This is indeed the case when $w(\Delta)$ is large enough. In our application, $u=\lambda a$, and $v=\lambda b$ with $\lambda \approx t^{1 / 3}$. Thus $w(\Delta)$ is of order $t^{1 / 3}$ which is large, and the triangle $\Delta$ is "small" compared to $T$.

Theorem 5.1: Assume $w(\Delta)$ is large enough $(w(\Delta) \geq 6)$. Then

$$
|| \mathbb{P} \cap \Delta\left|-\frac{6}{\pi^{2}} \Delta\right| \leq 30 \Delta \frac{\log w(\Delta)}{w(\Delta)} .
$$

Any given $x \in \Delta$ can be written uniquely as $x=\alpha(x) u+\beta(x) v$. Clearly, $\alpha(x)=x \cdot v^{\perp} / u \cdot v^{\perp}$, and $\int_{\Delta} \alpha(x) d x=\Delta / 3$.

Theorem 5.2: Assume $w(\Delta)$ is large enough $(w(\Delta) \geq 6)$. Then

$$
\left|\sum_{p \in \mathbb{P} \cap \Delta} \alpha(p)-\frac{6}{\pi^{2}} \int_{x \in \Delta} \alpha(x) d x\right|<30 \Delta \frac{\log w(\Delta)}{w(\Delta)}
$$

and

$$
\left|\sum_{p \in \mathbb{P} \cap \Delta} \beta(p)-\frac{6}{\pi^{2}} \int_{x \in \Delta} \beta(x) d x\right|<30 \Delta \frac{\log w(\Delta)}{w(\Delta)} .
$$

Notice that the estimate in both theorems is invariant under lattice preserving affine transformations. The proofs go via the classical method (see [HW79], for instance) and are therefore omitted. See also [BT04] for very similar proofs with the estimation depending on the lattice width of $\Delta$. 


\section{Proof of Theorem 4.1}

We assume $t=t_{1}=t_{2}$ and set $U=t a, V=t b$. For $x \in \mathbb{R}^{2}$, there is a unique representation $x=\bar{\alpha}(x) U+\bar{\beta}(x) V$.

We start with the upper bound. Let $x_{0}, \ldots, x_{n}$ be the sequence of vertices of a maximal lattice chain in $T$. So $m(T)=n$. The vectors $z_{i}=x_{i}-x_{i-1}$ all lie in $\mathbb{Z}^{2}$ and all belong to the cone pos $\{a, b\}$. Clearly

$$
\sum_{i=1}^{n} \bar{\alpha}\left(z_{i}\right) \leq 1 \quad \text { and } \quad \sum_{i=1}^{n} \bar{\beta}\left(z_{i}\right) \leq 1 .
$$

Define the norm (essentially an $\ell_{1}$ norm) $\|\cdot\|$ as follows:

$$
\|x\|=|\bar{\alpha}(x)|+|\bar{\beta}(x)| .
$$

Since the $z_{i}$ are non-parallel vectors from $\mathbb{Z}^{2} \cap \operatorname{pos}\{a, b\}$,

$$
\sum_{i=1}^{n}\left\|z_{i}\right\| \geq \sum\|p\|
$$

where the second sum is taken over the shortest (in $\|\cdot\|$ norm) $n$ primitive vectors in $\operatorname{pos}\{a, b\}$. The set of these shortest $n$ vectors from $\mathbb{P} \cap \operatorname{pos}\{a, b\}$ is exactly $\mathbb{P} \cap \Delta$, where $\Delta=\operatorname{conv}\{0, \lambda a, \lambda b\}$, for some suitable $\lambda>0$. (There is a little ambiguity in this definition when the side of $\Delta$ opposite to 0 contains many lattice points. This can be resolved in any way.)

The proof of the upper bound is based on choosing $\lambda$ so that $\sum_{\Delta \cap \mathbb{P}}\|p\|$ is almost equal to, but slightly larger than, 2 . In this case

$$
\sum_{i=1}^{n} \bar{\alpha}\left(z_{i}\right)+\sum_{i=1}^{n} \bar{\beta}\left(z_{i}\right) \geq \sum_{i=1}^{n}\left\|z_{i}\right\| \geq \sum_{p \in \Delta \cap \mathbb{P}}\|p\|>2,
$$

so $|\mathbb{P} \cap \Delta|>n=m(T)$, and we estimate $|\mathbb{P} \cap \Delta|$ using Theorem 5.1.

The computation is as follows. Setting $u=\lambda a, v=\lambda b$,

$$
\bar{\alpha}(x)=\frac{V^{\perp} \cdot x}{V^{\perp} \cdot U}=\frac{\lambda}{t} \frac{v^{\perp} \cdot x}{v^{\perp} \cdot u}=\frac{\lambda}{t} \alpha(x) .
$$

Write $\Delta_{0}$ for the triangle conv $\{0, a, b\}$ (and its area). We have $\Delta=\lambda^{2} \Delta_{0}$, $w(\Delta)=\lambda w\left(\Delta_{0}\right)$ and

$$
\int_{\Delta} \alpha(x) d x=\int_{\Delta} \beta(x) d x=\frac{1}{3} \Delta=\frac{1}{3} \lambda^{2} \Delta_{0} .
$$


By Theorem 5.2,

$$
\begin{aligned}
\sum_{p \in \Delta \cap \mathbb{P}} \bar{\alpha}(p) & =\frac{\lambda}{t} \sum_{p \in \Delta \cap \mathbb{P}} \alpha(p) \geq \frac{\lambda}{t}\left[\frac{6}{\pi^{2}} \frac{\lambda^{2} \Delta_{0}}{3}-30 \lambda^{2} \Delta_{0} \frac{\log w(\Delta)}{w(\Delta)}\right] \\
& =\frac{2 \lambda^{3} \Delta_{0}}{\pi^{2} t}\left(1-15 \pi^{2} \frac{\log w(\Delta)}{w(\Delta)}\right) .
\end{aligned}
$$

Set now

$$
\lambda=\sqrt[3]{\frac{\pi^{2} t}{2 \Delta_{0}}(1+\delta)}
$$

where $\delta>0$ will be soon specified. Now

$$
\lambda>\sqrt[3]{\frac{\pi^{2} t}{2 \Delta_{0}}}
$$

so, for large enough $t$,

$$
\frac{\log w(\Delta)}{w(\Delta)}=\frac{\log \lambda w\left(\Delta_{0}\right)}{\lambda w\left(\Delta_{0}\right)} \leq c_{1} t^{-1 / 3} \log t
$$

with a constant $c_{1}>0$ depending only on $\Delta_{0}$. Choose $\delta=30 \pi^{2} c_{1} t^{-1 / 3} \log t$. With this choice,

$$
\sum_{p \in \Delta \cap \mathbb{P}} \bar{\alpha}(p) \geq\left(1+30 \pi^{2} c_{1} t^{-1 / 3} \log t\right)\left(1-15 \pi^{2} c_{1} t^{-1 / 3} \log t\right)>1
$$

if $t$ is large enough. Similarly, $\sum_{p \in \mathbb{P} \cap \Delta} \bar{\beta}(p)>1$ and so $\sum_{p \in \mathbb{P} \cap \Delta}\|p\|>2$. Consequently,

$$
\begin{aligned}
m(T) & \leq|\Delta \cap \mathbb{P}|<\frac{6}{\pi^{2}} \Delta+30 \Delta \frac{\log w(\Delta)}{w(\Delta)} \\
& =\frac{6}{\pi^{2}} \Delta\left(1+5 \pi^{2} \frac{\log w(\Delta)}{w(\Delta)}\right) \\
& \leq \frac{6}{\pi^{2}}\left(\frac{\pi^{2} t}{2 \Delta_{0}}\right)^{2 / 3} \Delta_{0}(1+\delta)^{2 / 3}\left(1+5 \pi^{2} c_{1} t^{-1 / 3} \log t\right) \\
& =\frac{6}{(2 \pi)^{2 / 3}} T^{1 / 3}\left(1+O\left(t^{-1 / 3} \log t\right)\right) .
\end{aligned}
$$

Note that the implied constant depends on $\Delta_{0}$ only.

For the lower bound, choose $\lambda$ so that both $\sum_{p \in \mathbb{P} \cap \Delta} \bar{\alpha}(p), \sum_{p \in \mathbb{P} \cap \Delta} \bar{\beta}(p) \leq 1$ but almost equal to one. Then the primitive vectors in $\Delta$ can serve as edges of a convex lattice chain in $T$, so $m(T) \geq|\mathbb{P} \cap \Delta|$. For later reference we denote 
this convex lattice chain by $C(\Delta)$. The computation is similar to the previous one. We set

$$
\lambda=\sqrt[3]{\frac{\pi^{2} t}{2 \Delta_{0}}(1-\delta)} \text { and } \delta=\min \left(\frac{1}{2}, 15 \pi^{2} c_{2} t^{-1 / 3} \log t\right)
$$

where $c_{2}$ will be specified soon. Thus

$$
\lambda \geq \sqrt[3]{\frac{\pi^{2} t}{4 \Delta_{0}}}
$$

is of order $\sqrt[3]{t}$. Consequently

$$
\frac{\log w(\Delta)}{w(\Delta)} \leq c_{2} t^{-1 / 3} \log t
$$

with some large $c_{2}$ (depending only on $\Delta_{0}$ ) for all large enough $t$. This shows that $\delta<1 / 2$ if $t$ is large enough and so

$$
\delta=15 \pi^{2} c_{2} t^{-1 / 3} \log t
$$

We check that $\sum \bar{\alpha}(p) \leq 1$; indeed,

$$
\begin{aligned}
\sum_{p \in \Delta \cap \mathbb{P}} \bar{\alpha}(p) & =\frac{\lambda}{t} \sum_{p \in \Delta \cap \mathbb{P}} \alpha(p) \leq \frac{\lambda}{t}\left[\frac{6}{\pi^{2}} \frac{\Delta}{3}+30 \Delta \frac{\log w(\Delta)}{w(\Delta)}\right] \\
& =\frac{2 \lambda^{3} \Delta_{0}}{\pi^{2} t}\left(1+15 \pi^{2} \frac{\log w(\Delta)}{w(\Delta)}\right) \\
& =(1-\delta)\left(1+15 \pi^{2} c_{2} t^{-1 / 3} \log t\right)<1,
\end{aligned}
$$

and similarly for $\sum_{p \in \mathbb{P} \cap \Delta} \bar{\beta}(p) \leq 1$. Finally,

$$
\begin{aligned}
m(T) & \geq|\Delta \cap \mathbb{P}| \geq \frac{6}{\pi^{2}} \Delta-30 \Delta \frac{\log w(\Delta)}{w(\Delta)} \\
& \geq \frac{6}{(2 \pi)^{2 / 3}} T^{1 / 3}\left(1-O\left(t^{-1 / 3} \log t\right)\right) .
\end{aligned}
$$

Remark 6.1: The last proof contains the construction of an almost maximal lattice chain in $T$, namely $C(\Delta)$. The edges of this chain are the vectors in $\mathbb{P} \cap \Delta$. Its length is

$$
|\mathbb{P} \cap \Delta|=\frac{6}{(2 \pi)^{2 / 3}} T^{1 / 3}\left(1+O\left(t^{-1 / 3} \log t\right)\right) .
$$


The chain $C(\Delta)=\left(x_{0}, x_{1}, \ldots, x_{n}\right)$ almost connects the two special vertices, $p_{0}$ and $p_{2}$, of $T$ :

$$
\sum_{p \in \Delta \cap \mathbb{P}} p=\left(\sum_{p \in \Delta \cap \mathbb{P}} \bar{\alpha}(p)\right) U+\left(\sum_{p \in \Delta \cap \mathbb{P}} \bar{\beta}(p)\right) V
$$

Here, the coefficients of $U$ and $V$ are between $1-O\left(t^{-1 / 3} \log t\right)$ and 1 . So setting $x_{0}-p_{0}=\alpha_{0} a$ and $p_{2}-x_{n}=\beta_{0} b$ we have $\alpha_{0}, \beta_{0}=O\left(t^{2 / 3} \log t\right)$.

\section{Proof of Theorem 4.2}

Now $T$ is fixed and $Q_{t}$ is a maximal $\mathbb{Z}_{t}$-lattice chain within $T$. Let $\ell$ be a line, with fixed direction $d$, tangent to $Q_{t}$ at a vertex $p_{3}$. Let $q \in D(T)$ be the point where the tangent to $D(T)$ goes in direction $d$. Assume $\ell$ intersects $p_{0} p_{1}$ at $q_{1}$ and $p_{1} p_{2}$ at $q_{2}$. Let $T_{1}$ and $T_{2}$ be the triangles with vertices $p_{0}, q_{1}, p_{3}$ and $p_{3}, q_{2}, p_{2}$, resp. (Of course $\ell, p_{3}, q_{1}, q_{2}, T_{1}, T_{2}$ all depend on $t$.) See Figure 1 . Clearly,

$$
m^{*}\left(T, \mathbb{Z}_{t}\right)=m^{*}\left(T_{1}, \mathbb{Z}_{t}\right)+m^{*}\left(T_{2}, \mathbb{Z}_{t}\right)
$$

Choose convergent subsequences of $q_{1}, p_{3}, q_{2}$. Assuming none of the $p_{i}$ and $q_{j}$ coincides in the limit, we can apply the $\mathbb{Z}_{t}$ version of Theorem 4.1. This gives, after straightforward simplifications, that

$$
\sqrt[3]{T}=\sqrt[3]{T_{1}}+\sqrt[3]{T_{2}}
$$

where $T_{1}$ and $T_{2}$ denote the limiting triangles. In view of property (2.4) of the affine perimeter, this is possible if and only if $\ell$ (in the limit) is tangent to the parabola $D(T)$ at the point $q$. Thus $p_{3}$ tends to $q$.

If one of the triangles, say $T_{1}$, becomes degenerate, then one can use Andrew's estimate giving, again for the limiting triangles,

$$
\frac{6}{(2 \pi)^{2 / 3}} \sqrt[3]{T} \leq 20 \sqrt[3]{T_{1}}+\frac{6}{(2 \pi)^{2 / 3}} \sqrt[3]{T_{2}}
$$

But that is not possible since $T_{1}$, being degenerate, has area 0 and $T_{2}<T$. This finishes the proof of Theorem 4.2.

In the $\mathbb{Z}_{t}$ setting the chain $C(\Delta)$ becomes the $\mathbb{Z}_{t}$-lattice chain $C\left(\Delta, \mathbb{Z}_{t}\right)$ which is, of course, almost maximal and almost connects $p_{0}$ to $p_{2}$ within $T$. We show next that this chain, too, is very close to the parabola $D(T)$ : 
CLAIM 7.1: $\lim \operatorname{dist}\left(C\left(\Delta, \mathbb{Z}_{t}\right), D(T)\right)=0$.

Proof: For this proof we work in the $\mathbb{Z}^{2}$ setting and divide by $t$ in the end. Fix a vector $d$ on the segment $(a, b)$, that is, $d=(1-s) a+s b$ with $s \in(0,1)$ fixed, and let $\Delta(s)=\Delta \cap \operatorname{pos}(a, d)$. The tangent line to $C(\Delta)$ in direction $d$ goes through the point $q \in C(\Delta)$. Now

$$
q-p_{0}=\left(q-x_{0}\right)+\left(x_{0}-p_{0}\right)=\sum_{p \in \Delta(s) \cap \mathbb{P}} p+\left(x_{0}-p_{0}\right)
$$

Here $x_{0}-p_{0}$ is $O\left(t^{2 / 3} \log t\right) a$ from Remark 6.1. The sum of the vectors in $\Delta(s) \cap \mathbb{P}$ can be estimated the same way as for $\Delta \cap \mathbb{P}$ in Remark 6.1 and we get that

$$
\sum_{\Delta(s) \cap \mathbb{P}} p=s t(a+d)\left(1+O\left(t^{-1 / 3} \log t\right)\right) .
$$

The point $s t(a+d)$ is on the parabola $D(T)$ and the tangent there has direction $d$, as one can readily check. Thus $q-s t(a+d)$ is at most $O\left(t^{2 / 3} \log t\right)$. Dividing by $t$ we get the claim.

Remark 7.1: The primitive points are distributed evenly in a small triangle, so instead of summing them, we could take the integral of the vector $x$ in $\Delta$. In this case the triangle need not be small. With the previous notation,

$$
z(s)=\int_{\Delta(s)} x d x=\frac{1}{3} \Delta(s)(a+d)=\frac{1}{3} \Delta s((2-s) a+s b) .
$$

This is a curve, parametrized by $s$. It is very easy to see that this curve is exactly the special parabola inscribed in the triangle $\frac{1}{3} \Delta \operatorname{conv}\{0, a, a+b\}$. The tangent to this parabola at $z(s)$ is parallel with $d$.

Remark 7.2: The moral is that the maximal $\mathbb{Z}_{t}$-lattice chain in $\Delta$, and $C\left(\Delta, \mathbb{Z}_{t}\right)$ and $D(T)$ are all very close to each other. Further, $C\left(\Delta, \mathbb{Z}_{t}\right)$ is almost explicit; the curve $z(s)$ can be computed from $\Delta$. This is the idea behind the proof of Theorem 3.2.

\section{Proof of Theorem 2.1}

The proof consists of two parts. We show that the limsup of $t^{-2 / 3} \mid$ vert $Q_{t} \mid$, over a sequence of $\mathbb{Z}_{t}$-lattice convex curves $Q_{t} \subset U_{\varepsilon}(\Gamma)$, can only be slightly larger than $3(2 \pi)^{-2 / 3} A P(\Gamma)$. Secondly, we construct a sequence, $P_{t}$, of convex $\mathbb{Z}_{t}$-lattice polygons lying in $U_{\varepsilon}(\Gamma)$ with almost as many as $3(2 \pi)^{-2 / 3} t^{2 / 3} A P(\Gamma)$ vertices. 
Let $\eta$ be a small positive number, and choose $\varepsilon>0$ and unit vectors $d_{1}, \ldots, d_{k+1}$ for $\Gamma$ and $\eta$ according to Proposition 2.1. Let $Q_{t}(t \rightarrow \infty)$ be a sequence of convex $\mathbb{Z}_{t}$-lattice curves in $U_{\varepsilon}(\Gamma)$. We show that this sequence contains a subsequence, to be denoted by $Q_{\tau}$, such that

$$
\lim \sup \tau^{-2 / 3}\left|\operatorname{vert} Q_{\tau}\right| \leq \frac{3}{(2 \pi)^{2 / 3}}(A P(\Gamma)+3 \eta) .
$$

This shows what we need.

On each $Q_{t}$, the directions $d_{i}$ induce triangles $T_{i}(t)$ with vertices $z_{i}(t)$. We choose now a convergent subsequence $Q_{\tau}$ such that $\lim z_{i}(\tau)=x_{i}$ for each $i$. Let $Q \subset U_{\varepsilon}(\Gamma)$ be the limit of the $Q_{\tau}$. Clearly, $T_{i}(\tau)$ tends to a triangle $T_{i}$ for each $i$. Now

$$
A P(Q) \leq 2 \sum_{1}^{k} \sqrt[3]{T_{i}} \leq A P(\Gamma)+\eta
$$

Next we estimate $\left|\operatorname{vert} Q_{\tau}\right|$. We can apply Theorem $4.1, \mathbb{Z}_{t}$ version, in each $T_{i}(\tau)$ when $T_{i}$ is nondegenerate:

$$
m^{*}\left(T_{i}(\tau), \mathbb{Z}_{\tau}\right) \leq \frac{6}{(2 \pi)^{2 / 3}} \tau^{2 / 3}\left(\sqrt[3]{T_{i}(\tau)}+O\left(\tau^{-1 / 3} \log \tau\right)\right),
$$

where the constant in the 'big Oh' term depends on $T_{i}$ only. So for large enough $\tau$ (and nondegenerate $T_{i}$ ) we have

$$
m^{*}\left(T_{i}(\tau), \mathbb{Z}_{\tau}\right) \leq \frac{6}{(2 \pi)^{2 / 3}} \tau^{2 / 3}\left(\sqrt[3]{T_{i}}+\frac{\eta}{k}\right) .
$$

When $T_{i}$ is degenerate, Andrews' estimate works giving

$$
m^{*}\left(T_{i}(\tau), \mathbb{Z}_{\tau}\right) \leq 20 \tau^{2 / 3} \sqrt[3]{T_{i}(\tau)} \leq \tau^{2 / 3}\left(\sqrt[3]{T_{i}}+\frac{\eta}{k}\right),
$$

again for large enough $\tau$. Here $T_{i}=0$, since the triangle in question is degenerate. Thus for all $i$ and large $\tau$,

$$
m^{*}\left(T_{i}(\tau), \mathbb{Z}_{\tau}\right) \leq \frac{6}{(2 \pi)^{2 / 3}} \tau^{2 / 3}\left(\sqrt[3]{T_{i}}+\frac{\eta}{k}\right) .
$$

So we have, again for large enough $\tau$,

$$
\begin{aligned}
\left|\operatorname{vert} Q_{\tau}\right| & \leq \sum_{1}^{k} m^{*}\left(T_{i}(\tau), \mathbb{Z}_{\tau}\right) \leq \sum_{1}^{k} \frac{6}{(2 \pi)^{2 / 3}} \tau^{2 / 3}\left(\sqrt[3]{T_{i}}+\frac{\eta}{k}\right) \\
& \leq \frac{3}{(2 \pi)^{2 / 3}} \tau^{2 / 3} \sum_{1}^{k} 2\left(\sqrt[3]{T_{i}}+\frac{\eta}{k}\right) \leq \frac{6}{(2 \pi)^{2 / 3}} \tau^{2 / 3}(A P(\Gamma)+3 \eta) .
\end{aligned}
$$


Next comes the construction of $P_{t}$. Assume $\varepsilon>0$ is small. We will find a sequence of $\mathbb{Z}_{t}$-lattice polygons $P_{t}$ in $U_{\varepsilon}(\Gamma)$ such that $P_{t}$ has at least $3(2 \pi)^{-2 / 3} t^{2 / 3}(A P(\Gamma)-2 \varepsilon)$ vertices, provided $t$ is large enough.

Choose points $x_{i} \in \Gamma$, tangent lines $\ell_{i}$, so that the triangles $T_{i}$ all lie in $U_{\varepsilon / 2}(\Gamma)$. We assume, rather for convenience than necessity, that the $\ell_{i}$ have irrational slopes. By the properties of the affine perimeter,

$$
2 \sum_{1}^{k} \sqrt[3]{T_{i}} \geq A P(\Gamma) .
$$

For each $i$ there is a $\mathbb{Z}_{t}$-lattice square, of side length $1 / t$, containing $x_{i}$. Move $\ell_{i}$ to a parallel position, $\ell_{i}(t)$, that contains a vertex, $z_{i}(t)$, of this square. Here, $z_{i}(t) \in \mathbb{Z}_{t}$ is chosen so that the whole square and $\Gamma$ lie on the same side of $\ell_{i}(t)$. Replace each $x_{i}$ by $z_{i}(t) \in \mathbb{Z}_{t}$ and each triangle $T_{i}$ by the corresponding $T_{i}(t)$. Note that the $z_{i}(t)$ are in convex position.

Recall that the proof of the lower bound of Theorem 4.1 produced an almost maximal $\mathbb{Z}^{2}$-lattice chain $C(\Delta)$ in the triangle $\Delta$. This gives in the $\mathbb{Z}_{t}$-setting an almost maximal $\mathbb{Z}_{t}$-lattice chain $C\left(T_{i}(t), \mathbb{Z}_{t}\right)$ in $T_{i}(t)$. Fix $i$ now and let $z_{i}(t), y_{0}, y_{1}, \ldots, y_{n}, z_{i+1}(t)$ be the vertices of $C\left(T_{i}(t), \mathbb{Z}_{t}\right)$. Note that $y_{j}-y_{j-1}$ is in $\mathbb{Z}_{t}$ but $y_{j}$ may not be.

We show now how this chain can be changed a little so that it is an almost maximal $\mathbb{Z}_{t}$-lattice chain within $T_{i}(t)$ with all of its vertices in $\mathbb{Z}_{t}$. To this end note first that $v=\left(y_{0}-z_{i}(t)\right)+\left(z_{i+1}(t)-y_{n}\right)$ is in $\mathbb{Z}_{t}$. Also, the slope of $v$ is between the slopes of two consecutive edges of the chain $C\left(T_{i}(t), \mathbb{Z}_{t}\right)$, say the $j$ th and the $(j+1)$ st. Then the vectors

$$
y_{1}-y_{0}, y_{2}-y_{1}, \ldots, y_{j}-y_{j-1}, v, y_{j+1}-y_{j}, \ldots, y_{n}-y_{n-1}
$$

form the edges, in this order, of a chain from $z_{i}(t)$ to $z_{i+1}(t)$ within $T_{i}(t)$ with all vertices in $\mathbb{Z}_{t}$. Let $C_{i}(t)$ denote this chain. It has at least as many edges as the original chain had.

Since $T_{i} \subset U_{\varepsilon / 2}(\Gamma)$, both $T_{i}(t)$ and $C_{i}(T)$ lie in $U_{\varepsilon}(\Gamma)$ if $t$ is large enough. Thus the union of the $C_{i}(t)$ forms a convex $\mathbb{Z}_{t}$-lattice curve $P_{t}$ in $U_{\varepsilon}(\Gamma)$. The construction is now finished. We have to bound the number of vertices of $P_{t}$ from below.

The number of edges on $C_{i}(t)$ is at least as large as on $C\left(T_{i}(t), \mathbb{Z}_{t}\right)$ which is, by Theorem 4.1 , at least

$$
\frac{6}{(2 \pi)^{2 / 3}} \sqrt[3]{T_{i}(t)}\left(1-O\left(t^{-1 / 3} \log t\right)\right),
$$


provided $T_{i}$ is nondegenerate. Note that for such a $T_{i}$,

$$
\sqrt[3]{T_{i}(t)}\left(1-O\left(t^{-1 / 3} \log t\right)\right) \geq \sqrt[3]{T_{i}}-\varepsilon / k
$$

if $t$ is large enough. For degenerate $T_{i}$, we use the trivial estimate saying that $C_{i}(t)$ has at least $t^{2 / 3} \sqrt[3]{T_{i}}=0$ edges. So we have

$$
\begin{aligned}
\mid \text { vert } P_{t} \mid & \geq \sum_{1}^{k} \frac{6}{(2 \pi)^{2 / 3}} t^{2 / 3}\left(\sqrt[3]{T_{i}}-\frac{\varepsilon}{k}\right) \\
& \geq \frac{3}{(2 \pi)^{2 / 3}} t^{2 / 3} \sum_{1}^{k} 2\left(\sqrt[3]{T_{i}}-\frac{\varepsilon}{k}\right) \\
& \geq \frac{3}{(2 \pi)^{2 / 3}} t^{2 / 3}(A P(\Gamma)-2 \varepsilon)
\end{aligned}
$$

Remark 8.1: $\quad C_{i}(t)$ is made up of primitive vectors in $\Delta_{i}(t)$ plus possibly one more vector, which we ignore as it is short. So the edges of $P_{t}$ are essentially the primitive vectors in $\bigcup \Delta_{i}(t)$. In the proof of Theorem 3.2 , we will need to show that the set $\bigcup \Delta_{i}(t)$ has nice properties.

\section{Proof of Theorems 1.1 and 1.2}

Let $Q_{t}$ be any maximizer in the definition of $m\left(K, \mathbb{Z}_{t}\right)$. Choose a subsequence $Q_{t}^{\prime}$ from it. We show that it contains a subsequence, to be denoted by $Q_{\tau}$, with $\tau \rightarrow \infty$ such that

$$
\lim \tau^{-2 / 3}\left|\operatorname{vert} Q_{\tau}\right|=\frac{3}{(2 \pi)^{2 / 3}} A(K)
$$

and

$$
\lim \operatorname{dist}\left(Q_{\tau}, K_{0}\right)=0
$$

This will prove both theorems.

The proof is simple and is based on Theorem 2.1. Choose a convergent subsequence $Q_{\tau}$ from $Q_{t}^{\prime}$, and let $S$ be the limit of $Q_{\tau}$. Clearly, $S \subset K$ and $S \in \mathcal{C}$. By Theorem 2.1,

$$
\lim \tau^{-2 / 3}\left|\operatorname{vert} Q_{\tau}\right|=\frac{3}{(2 \pi)^{2 / 3}} A P(S)
$$

By the definition of $A(K), A P(S) \leq A(K)$. Assume $A P(S)<A(K)$. Then $A P(S)+\eta<A(K)$ with some positive $\eta$. A slightly shrunken homothetic copy of $K_{0}, K_{0}^{\prime}$ say, can be placed in $K$ so that $U_{\varepsilon}\left(\partial K_{0}^{\prime}\right) \subset K$ for some positive $\varepsilon$, and 
$A P(S)+\eta / 2<A P\left(K_{0}^{\prime}\right)$. Now Theorem 2.1 implies the existence of a convex $\mathbb{Z}_{t}$-lattice polygon $P_{\tau}$ in $U_{\varepsilon}\left(K_{0}^{\prime}\right) \subset K$ with

$$
\mid \text { vert } P_{\tau} \mid>\frac{3}{(2 \pi)^{2 / 3}} \tau^{2 / 3}(A P(S)+\eta / 4) \text {. }
$$

This contradicts the maximality of $Q_{\tau}$. Then $A P(S)=A(K)$, and $S=K_{0}$ follows from the unicity of $K_{0}$.

\section{Proof of Theorem 3.2}

We assume first that $K$ is a convex polygon. Then $K_{0}$ is tangent to edges $E_{1}, \ldots, E_{k}$ of $K$ at points $p_{1}, \ldots, p_{k}$. We assume that the boundary of $K$ is exactly $\bigcup E_{i}$ (since we can delete the edges not touched by $K_{0}$ ). The vertex $v_{i}$ is the common endpoint of edges $E_{i}$ and $E_{i+1}$. The outer angle at $v_{i}$ is $\phi_{i}>0$. Set $T_{i}=\operatorname{conv}\left\{p_{i}, v_{i}, p_{i+1}\right\}$. Then $A P(K)=2 \sum_{1}^{k} \sqrt[3]{T_{i}}$ is the solution of the maximization problem

$$
\max 2 \sum_{1}^{k} \sqrt[3]{x_{i}\left(e_{i+1}-x_{i+1}\right) \sin \phi_{i}}
$$

where $e_{i}=\left\|E_{i}\right\|$ and $x_{i}=\left\|p_{i}-v_{i}\right\|$; see Figure 2 .

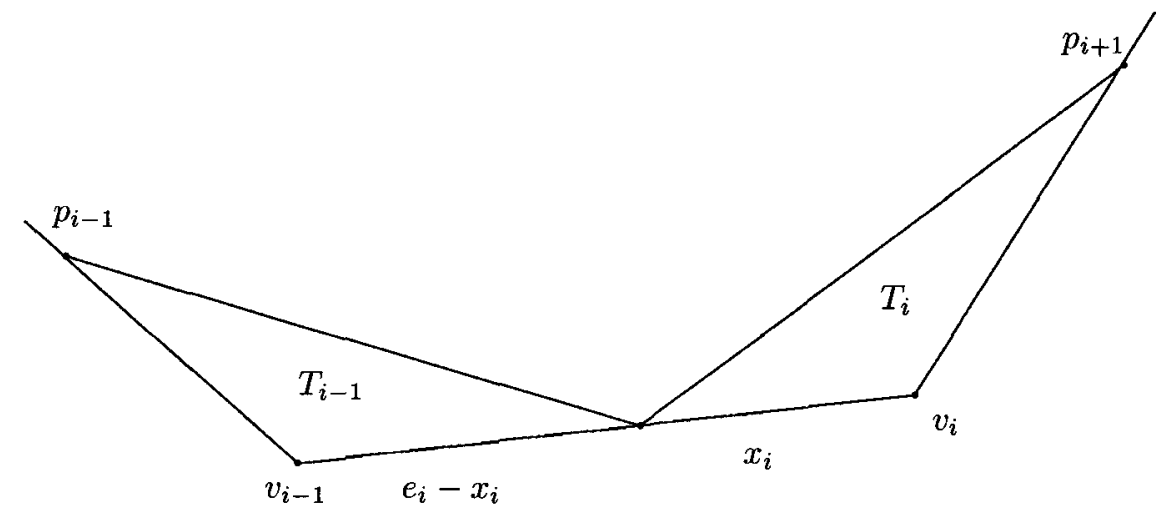

Figure 2.

The solution is unique (by Theorem 3.1). So taking derivatives we get the necessary conditions for the extremum. They can be expressed as

$$
\frac{x_{i}}{\sqrt[3]{T_{i}}}=\frac{e_{i}-x_{i}}{\sqrt[3]{T_{i-1}}}
$$


Define now the triangle

$$
\Delta_{i}=\frac{1}{\sqrt[3]{T_{i}}} \operatorname{conv}\left\{0, v_{i}-p_{i}, p_{i+1}-v_{i}\right\}
$$

So $T_{i}$ determines $\Delta_{i}$. Conversely, $\Delta_{i}$ determines $T_{i}$ uniquely (up to translation) in the following way. If $\Delta_{i}=\operatorname{conv}\left\{0, a_{i}, b_{i}\right\}$, then

$$
\operatorname{conv}\left\{0, \Delta_{i} a_{i}, \Delta_{i}\left(a_{i}+b_{i}\right)\right\}
$$

is a translated copy of $T_{i}$.

Each $\Delta_{i}$ has 0 as a vertex. Note that $\Delta_{i}$ and $\Delta_{i-1}$ share an edge. Namely, the edge going in direction $v_{i}-v_{i-1}$ has length $x_{i} / \sqrt[3]{T_{i}}$ in $\Delta_{i}$ and $\left(e_{i}-x_{i}\right) / \sqrt[3]{T_{i-1}}$ in $\Delta_{i-1}$. They are equal, according to the necessary conditions for the extremum. CLAIM 10.1: $\bigcup_{1}^{k} \Delta_{i}$ is a convex set.

Proof: We have to see that the angle, say $\psi$, at the common vertex of $\Delta_{i} \cup \Delta_{i-1}$ is less than $\pi$. To have simpler notation we assume that $i=2$ and $\Delta_{1}=$ $\operatorname{conv}\left\{0, a_{1}, b_{1}\right\}, \Delta_{2}=\operatorname{conv}\left\{0, a_{2}, b_{2}\right\}$. Here, as we have seen, $b_{1}=a_{2}$.

If $\phi_{1}+\phi_{2} \geq \pi$, then $\psi<\pi$ follows immediately. So assume $\phi_{1}+\phi_{2}<\pi$. Then $\psi<\pi$ if and only if $\Delta_{1}+\Delta_{2}>\Delta$, where $\Delta=\left\{0, a_{1}, b_{2}\right\}$.

As the statement is invariant under linear transformations, we may assume that $a_{1}=(1,0)$ and $b_{2}=(0,1)$, and write $b_{1}=a_{2}=(r \sin \alpha, r \cos \alpha)$ with $\alpha \in(0, \pi / 2)$ and $r>0$. With this notation our target is to show that

$$
r(\sin \alpha+\cos \alpha)>1 \text {. }
$$

Now $T_{1}$ and $T_{2}$ are determined by $\Delta_{1}$ and $\Delta_{2}$ (see Figure 3 for notation). It is clear that $A=\left(r^{2} / 2\right)(\sin \alpha+\cos \alpha) \cos \alpha$ and $B=\left(r^{2} / 2\right)(\sin \alpha+\cos \alpha) \sin \alpha$. The special parabola arc within the triangle $T_{0}=\operatorname{conv}\left\{p_{1}, p_{0}, p_{3}\right\}$ connecting $p_{1}$ and $p_{3}$ must intersect the segment $\left[v_{1}, v_{2}\right]$, as otherwise replacing $T_{1}$ and $T_{2}$ by $T_{0}$ would increase the affine perimeter of $K$. It is not difficult to check that this happens if and only if $A B>\left(r^{2} / 4\right) \sin \alpha \cos \alpha$. As

$$
A B=\left(r^{4} / 4\right)(\sin \alpha+\cos \alpha)^{2} \sin \alpha \cos \alpha
$$

we get

$$
r^{2}(\sin \alpha+\cos \alpha)^{2}>1
$$

which is equivalent to our target. 


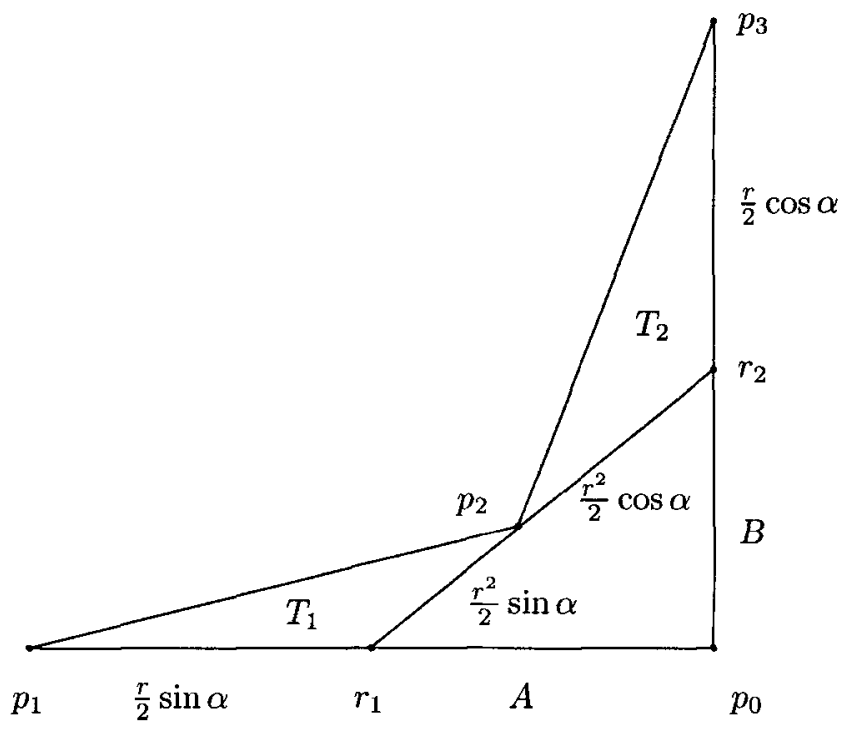

Figure 3.

We show now that the convex set $\bigcup_{1}^{k} \Delta_{i}$ has its barycenter at 0 . Remark 7.1 shows that

$$
\int_{\Delta_{i}} x d x=\frac{1}{3} \Delta_{i}\left(a_{i}+b_{i}\right)=p_{i+1}-p_{i} .
$$

This implies immediately that indeed $\int_{\cup \Delta_{i}} x d x=\sum_{1}^{k}\left(p_{i+1}-p_{i}\right)=0$.

We are almost finished with the proof. Define $C$ to be the copy of $\cup \Delta_{i}$ rotated around the origin, in clockwise direction, by $\pi / 2$. Let $u$ be a unit vector, and let $z(u)$ be the unique point with outer normal $u$ on the boundary of $K_{0}$. What's the radius of curvature, $R(u)$, of $K_{0}$ at $z(u)$ ? This is, by definition, the limit, as $v \rightarrow u$, of the length of the arc on $\partial K_{0}$ between $z(u)$ and $z(v)$ divided by the angle between $u, v \in S^{1}$. We may assume both directions $u, v$ lie in the triangle $\Delta_{i}^{\perp}$. Define $\Delta(u, v)=\operatorname{pos}\{u, v\} \cap \Delta_{i}^{\perp} ;$ then

$$
\int_{\Delta(u, v)} x d x \approx \frac{1}{3} \rho_{C}(u)^{3}\|u-v\| u,
$$

and so $R(u)=\frac{1}{3} \rho_{C}(u)^{3}$. This proves the first half of Theorem 3.2 in the case when $K$ is a polygon. 
Assume now $K \in \mathbb{R}^{2}$ is arbitrary, and let $P_{n}$ be a sequence of convex polygons tending to $F(K)=K_{0}$ with $K_{0} \subset P_{n}$. Then $F\left(P_{n}\right) \rightarrow K_{0}$ as well. Also, by the previous argument, $F\left(P_{n}\right)=G\left(C_{n}\right)$ with a unique $C_{n} \in \mathcal{C}_{0}$ and $R_{F\left(P_{n}\right)}(u)=$ $\rho_{C_{n}}^{3}(u) / 3$. By property $(3.3), R_{F\left(P_{n}\right)}(u)$ is bounded. Then one can choose a convergent subsequence from $C_{n}$ tending to $C \in \mathcal{C}_{0}$. It is easy to see that not only the subsequence but the whole $C_{n}$ tends to $C$ implying, in turn, that $\rho_{C_{n}} \rightarrow \rho_{C}$, and so $\lim R_{F\left(P_{n}\right)}(u)=\rho_{C}^{3}(u) / 3$ for each $u$. It follows now that $R_{K_{0}}(u)=\rho_{C}^{3}(u) / 3$.

The second half of the theorem is easy: $G(C)=C^{*} \in \mathcal{C}$ clearly. Choose a dense enough set of directions $d_{1}, \ldots, d_{n+1}$ and consider the induced triangles, $T_{i}$, on $C^{*}$. The corresponding "small triangles" $\Delta_{i}$ are very close to $C^{\perp} \cap \operatorname{pos}\left\{d_{i}, d_{i+1}\right\}$, where $C^{\perp}$ is a copy of $C$ rotated by $\pi / 2$ (in anticlockwise direction) since $R_{C^{*}}(u)=\rho_{C}^{3}(u) / 3$. Then the rotated copy of $\bigcup \Delta_{i}$ is very close to $C$.

\section{Proof of Corollary 3.2}

Let $L: \mathbb{R}^{2} \rightarrow \mathbb{R}^{2}$ be a nondegenerate linear map. For $K \in \mathcal{C}, F(L K)=L F(K)$. Assume now that $C \in \mathcal{C}_{0}$ is the convex set whose existence is guaranteed by Theorem 3.2. We claim that $L C$ is the convex set corresponding to $L K$. It suffices to check this when $K$ is a convex polygon. The proof of Theorem 3.2 shows that $\bigcup \Delta_{i}$ is a convex polygon with each $\Delta_{i}$ a well-defined triangle. As $\int_{\Delta_{i}(s)} x d x$ describes the special parabola in $T_{i}, \int_{L \Delta_{i}(s)} x d x$ describes the special parabola in $L T_{i}$. As $C$ is a rotated copy of $\bigcup \Delta_{i}, L C$ is also a rotated copy of $\bigcup L \Delta_{i}$. This proves the claim.

Given $K \in \mathcal{C}$ and the corresponding $C \in \mathcal{C}_{0}$, choose a linear transformation $L: \mathbb{R}^{2} \rightarrow \mathbb{R}^{2}$ that carries $C$ into isotropic position. This means (see [KLS]) that $b(C)=0$ and the matrix of inertia about $0, \frac{1}{\operatorname{Area} C} \int_{x \in C} x x^{T} d x$, is the identity matrix. Kannan, Lovász and Simonovits [KLS] prove that, with this positioning, the inscribed and circumscribed ellipses of $C$ can be taken to be circles $B(r), B(R)$, centered at the origin, with $R / r \leq 2$. So, we may take $r=1$ and then $R \leq 2$.

In this position the radial function $\rho_{L C}(u)$ satisfies

$$
1 \leq \rho_{L C}(u) \leq 2,
$$

and the radius of curvature of $F(L K)$ satisfies the required inequalities. 


\section{References}

[An63] G. E. Andrews, A lower bound for the volumes of strictly convex bodies with many boundary points, Transactions of the American Mathematical Society 106 (1963), 270-279.

[Bá95] I. Bárány, The limit shape of convex lattice polygons, Discrete and Computational Geometry 13 (1995), 279-295.

[Bá97] I. Bárány, Affine perimeter and limit shape, Journal für die reine und angewandte Mathematik 484 (1997), 71-84.

[Bá99] I. Bárány, Sylvester's question: The probability that $n$ points are in convex position, The Annals of Probability 27 (1999), 2020 2034.

[BT04] I. Bárány and N. Tokushige, The minimum area convex lattice $n$-gon, Combinatorica 24 (2004), 171-185.

[B123] W. Blaschke, Vorlesungen über Differenzialgeometrie II. Affine Differenzialgeometrie, Springer, Berlin, 1993.

[HW79] G. H. Hardy and E. M. Wright, An Introduction to the Theory of Numbers, Clarendon Press, Oxford, 1979.

[Ja25] V. Jarník, Über Gitterpunkte und konvex Kurven, Mathematische Zeitschrift 2 (1925), 500-518.

[KLS] R. Kannan, L. Lovász and M. Simonovits, Isoperimetric problems for convex bodies and a localization lemma, Discrete and Computational Geometry 13 (1995), 541 -559.

[Lu91] E. Lutwak, Extended affine surface area, Advances in Mathematics 85 (1991), $39-68$.

[Ra93] S. Rabinowitz, $O\left(n^{3}\right)$ bounds for the area of a convex lattice $n$-gon, Geombinatorics 2 (1993), 85-89.

[RS63] A. Rényi and R. Sulanke, Über die konvexe Hülle von $n$ zufällig gewählten Punkten, Zeitschrift für Wahrscheinlichkeitstheorie und Verwandte Gebiete 2 (1963), 75-84.

[Sch93] R. Schneider, Convex Bodies: The Brunn-Minkowski Theory, Cambridge University Press, Cambridge, 1993.

[Ve94] A. M. Vershik, The limit shape for convex lattice polygons and related topics, Functional Analysis and its Applications 28 (1994), 16-25 (in Russian) and Functional Analysis and its Applications 28 (1994), 13-20 (English translation).

[Žu95] J. Žunić, On the maximal number of edges of convex digital polygons included in the $m \times m$ grid, Journal of Combinatorial Theory. Series A 69 (1995), 358368. 
[Žu98] J. Žunić, Limit shape of convex lattice polytopes having minimal $L_{\infty}$ diameter w.r.t. the number of their vertices, Discrete Mathematics 187 (1998), 245254. 